\title{
Explaining Time-Table-Edge-Finding Propagation for the Cumulative Resource Constraint
}

\author{
Andreas Schutt Thibaut Feydy Peter J. Stuckey \\ Optimisation Research Group, National ICT Australia, and Department of Computing and \\ Information Systems, The University of Melbourne, Victoria 3010, Australia \\ $\{$ andreas.schutt, thibaut.feydy, peter.stuckey\}@nicta.com.au
}

\begin{abstract}
Cumulative resource constraints can model scarce resources in scheduling problems or a dimension in packing and cutting problems. In order to efficiently solve such problems with a constraint programming solver, it is important to have strong and fast propagators for cumulative resource constraints. One such propagator is the recently developed time-table-edge-finding propagator, which considers the current resource profile during the edge-finding propagation. Recently, lazy clause generation solvers, i.e., constraint programming solvers incorporating nogood learning, have proved to be excellent at solving scheduling and cutting problems. For such solvers, concise and accurate explanations of the reasons for propagation are essential for strong nogood learning. In this paper, we develop the first explaining version of time-table-edge-finding propagation and show preliminary results on resourceconstrained project scheduling problems from various standard benchmark suites. On the standard benchmark suite PSPLib, we were able to close one open instance and to improve the lower bound of about $60 \%$ of the remaining open instances. Moreover, 6 of those instances were closed.
\end{abstract}

\section{Introduction}

A cumulative resource constraint models the relationship between a scarce resource and activities requiring some part of the resource capacity for their execution. Resources can be workers, processors, water, electricity, or, even, a dimension in a packing and cutting problem. Due to its relevance in many industrial scheduling and placement problems, it is important to have strong and fast propagation techniques in constraint programming (CP) solvers that detect inconsistencies early and remove many invalid values from the domains of the variables involved. Moreover, when using CP solvers that incorporate "fine-grained" nogood learning it is also important that each inconsistency and each 


\section{A}
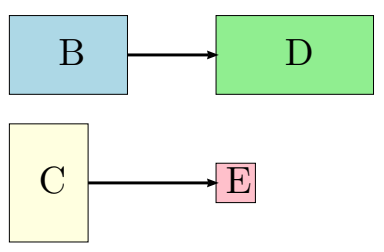

Figure 1: Five activities with precedence relations.

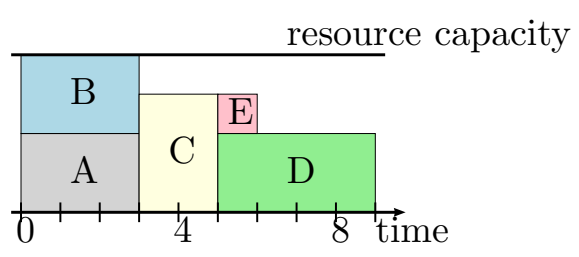

Figure 2: A possible schedule of the activities.

value removal from a domain is explained in such a way that the full strength of nogood learning is exploited.

In this paper, we consider renewable resources, i.e., resources with a constant resource capacity over time, and non-preemptive activities, i.e., whose execution cannot be interrupted, with fixed processing times and resource usages. In this work, we develop explanations for the time-table-edge-finding ( $\mathrm{TTEF}$ ) propagator [34] for use in lazy clause generation (LCG) solvers [22, 9].

Example 1.1. Consider a simple cumulative resource scheduling problem. There are 5 activities A, B, C, D, and E to be executed before time period 10. The activities have processing times $3,3,2,4$, and 1 , respectively, with each activity requiring $2,2,3,2$, and 1 units of resource, respectively. There is a resource capacity of 4 . Assume further that there are precedence constraints: activity B must finish before activity D begins, written $B \ll D$, and similarly $C \ll E$. Figure 1 shows the five activities and precedence relations, while Fig. 2 shows a possible schedule, where the start times are: 0, 0, 3, 5, and 5 respectively.

In CP solvers, a cumulative resource constraint can be modelled by a decomposition or, more successfully, by the global constraint cumulative [2]. Since the introduction of this global constraint, a great deal of research has investigated stronger and faster propagation techniques. These include time-table [2], (extended) edge-finding [21, 33], not-first/not-last [21, 25], and energetic-reasoning propagation [4, 6. Time-table propagation is usually superior for highly disjunctive problems, i.e., in which only some activities can run concurrently, while (extended) edge-finding, not-first/not-last, and energetic reasoning are more appropriate for highly cumulative problems, i.e., in which many activities can run concurrently. [4] The reader is referred to [6] for a detailed comparison of these techniques.

Vilim [34] recently developed TTEF propagation which combines the time-table and (extended) edge-finding propagation in order to perform stronger propagation while having a low runtime overhead. Vilim [34] shows that on a range of highly disjunctive open resource-constrained project scheduling problems from the well-established benchmark 
library PSPLib TTEF propagation can generate lower bounds on the project deadline (makespan) that are superior to those found by previous methods. He uses a CP solver without nogood learning. This result, and the success of LCG on such problems, motivated us to study whether an explaining version of this propagation yields an improvement in performance for LCG solvers.

In general, nogood learning is a resolution step that infers redundant constraints, called nogoods, given an inconsistent solution state. These nogoods are permanently or temporarily added to the initial constraint system in order to reduce the search space and/or to guide the search. Moreover, they can be used to short circuit propagation. How this resolution step is performed is dependent on the underlying system.

LCG solvers employ a "fine-grained" nogood learning system that mimics the learning of modern Boolean satisfiability (SAT) solvers (see e.g. [20]). In order to create a strong nogood, it is necessary that each inconsistency and value removal is explained concisely and in the most general way possible. For LCG solvers, we have previously developed explanations for time-table and (extended) edge-finding propagation [27]. Moreover, for time-table propagation we have also considered the case when processing times, resource usages, and resource capacity are variable [24]. Explanations for the time-table propagator were successfully applied on resource-constraint project scheduling problems [27, 29] and carpet cutting [28] where in both cases the state-of-the-art of exact solution methods were substantially improved. The explanations defined here are similar to the step-wise ones for the (extended) edge-finding propagation in [27], but there we do not consider the resource profile and are more complex. Moreover, the proposed explanations for edge-finding propagation in [27] has never been implemented.

Explanations for the propagation of the cumulative constraint have also been proposed for the PaLM [14, 13] and SCIP [1, 7, 12] frameworks. In the PaLM framework, explanations are only considered for time-table propagation, while the SCIP framework additionally provides explanations for energetic reasoning propagation and a restricted version of edge-finding propagation. Neither framework consider bounds widening in order to generalise these explanations as we do in this paper. Other related works include [32, which presents explanations for different propagation techniques for problems only involving disjunctive resources, i.e., cumulative resources with unary resource capacity, and generalised nogoods [15]. A detailed comparison of explanations for the propagation of cumulative resource constraints in LCG solvers can be found in [24].

In this paper we develop explanations for the TTEF cumulative propagator in LCG solvers. The explaining TTEF propagation is then compared with the explaining timetable propagation from [27] in the LCG solver on RCPSP using the reengineered LCG solver [9] which was also used for the experiments presented in [27].

\section{Cumulative Resource Scheduling}

In cumulative resource scheduling, a set of (non-preemptive) activities $\mathcal{V}$ and one cumulative resource with a (constant) resource capacity $R$ is given where an activity $i$ is

\footnotetext{
${ }^{1}$ See http://129.187.106.231/psplib/
} 
specified by its start time $S_{i}$, its processing time $p_{i}$, its resource usage $r_{i}$, and its energy $e_{i}:=p_{i} \cdot r_{i}$. In this paper we assume each $S_{i}$ is an integer variable and all others are assumed to be integer constants. Further, we define $e^{s} t_{i}\left(e c t_{i}\right)$ and $l s t_{i}\left(l c t_{i}\right)$ as the earliest and latest start (completion) time of $i$.

In this setting. the cumulative resource scheduling problem is defined as a constraint satisfaction problem that is characterised by the set of activities $\mathcal{V}$ and a cumulative resource with resource capacity $R$. The goal is to find a solution that assigns values from the domain to the start time variables $S_{i}(i \in \mathcal{V})$, so that the following conditions are satisfied.

$$
\begin{array}{lr}
\text { est }_{i} \leq S_{i} \leq l s t_{i}, & \forall i \in \mathcal{V} \\
\sum_{i \in \mathcal{V}: \tau \in\left[S_{i}, S_{i}+p_{i}\right)} r_{i} \leq R & \forall \tau
\end{array}
$$

where $\tau$ ranges over the time periods considered. Note that this problem is NP-hard [5].

We shall tackle problems including cumulative resource scheduling using $\mathrm{CP}$ with nogood learning. In a CP solver, each variable $S_{i}, i \in \mathcal{V}$ has an initial domain of possible values $D^{0}\left(S_{i}\right)$ which is initially $\left[e s t_{i}, l s t_{i}\right]$. The solver maintains a current domain $D$ for all variables. CP search interleaves propagation with search. The constraints are represented by propagators that, given the current domain $D$, creates a new smaller domain $D^{\prime}$ by eliminating infeasible values. The current lower and upper bound of the domain $D\left(S_{i}\right)$ are denoted by $l b\left(S_{i}\right)$ and $u b\left(S_{i}\right)$, respectively. For more details on CP see e.g. [23].

For a learning solver we also represent the domain of each variable $S_{i}$ using Boolean variables $\llbracket S_{i} \leq v \rrbracket, e s t_{i} \leq v<l s t_{i}$. These are used to track the reasons for propagation and generate nogoods. For more details see [22]. We use the notation $\llbracket v \leq S_{i} \rrbracket$, est $_{i}<$ $v \leq l s t_{i}$ as shorthand for $\neg \llbracket S_{i} \leq v-1 \rrbracket$, and treat $\llbracket v \leq S_{i} \rrbracket, v \leq e s t_{i}$ and $\llbracket S_{i} \leq v \rrbracket, v \geq l s t_{i}$ as synonyms for true. Propagators in a learning solver must explain each reduction in the domain by building a clausal explanation using these Boolean variables.

Optimisation problems are typically solved in $\mathrm{CP}$ via branch and bound. Given an objective $o b j$ which is to be minimised, when a solution is found with objective value $o$, a new constraint $o b j<o$ is posted to enforce that we only look for better solutions in the subsequent search.

\section{TTEF Propagation}

In this section we develop explanations for TTEF propagation. For a more detailed description about TTEF propagation the reader is referred to [34].

TTEF propagation splits the treatment of activities into a fixed and free part. The former results from the activities' compulsory part whereas the latter is the remainder. The fixed part of an activity $i$ is characterised by the length of its compulsory part $p_{i}^{T T}:=$ $\max \left(0, e c t_{i}-l s t_{i}\right)$ and its fixed energy $e_{i}^{T T}:=r_{i} \cdot p_{i}^{T T}$. The free part has a processing time $p_{i}^{E F}:=p_{i}-p_{i}^{T T}$ and a free energy of $e_{i}^{E F}:=e_{i}-e_{i}^{E F}$. Let $\mathcal{V}^{E F}$ be the set of 


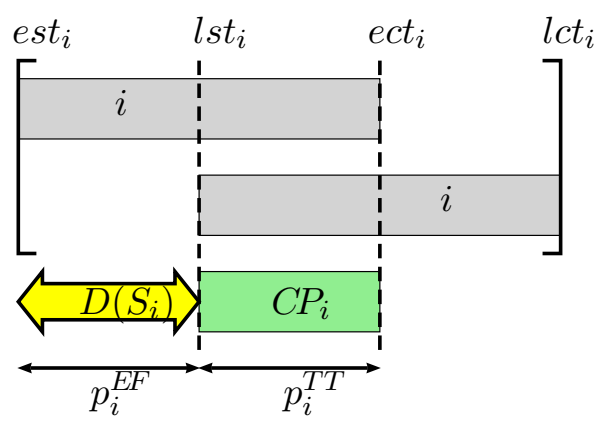

Figure 3: A diagram illustrating an activity $i$ when started at $e s t_{i}$ or $l s t_{i}$, and its possible range of start times, as well as the compulsory part $C P_{i}$, and the fixed and free parts of the processing time.

activities with a non-empty free part $\left\{i \in \mathcal{V} \mid p_{i}^{E F}>0\right\}$. An illustration of this is shown in Figure 3 .

TTEF propagation reasons about the energy available from the resource and energy required for the execution of activities in specific time windows. The start and end times of these windows are determined by the earliest start and the latest completion times of activities $i \in \mathcal{V}^{E F}$. These time windows [begin, end) are characterised by the so-called task intervals $\mathcal{V}^{E F}(a, b):=\left\{i \in \mathcal{V}^{E F} \mid e^{e s t_{a}} \leq\right.$ est $\left._{i} \wedge l c t_{i} \leq l c t_{b}\right\}$ where $a, b \in \mathcal{V}^{E F}$, begin $:=e s t_{a}$, and $e n d:=l_{c} t_{b}$.

It is not only the free energy of activities in the task interval $\mathcal{V}^{E F}(a, b)$ that is considered, but also the energy resulting from the compulsory parts in the time win-

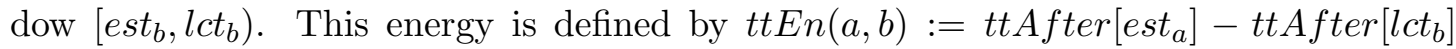
where $t$ After $[\tau]:=\sum_{t \geq \tau} \sum_{i \in \mathcal{V}: l s t_{i} \leq t<e c t_{i}} r_{i}$.

Furthermore, we also consider activities $i \in \mathcal{V} \backslash \mathcal{V}^{E F}(a, b)$ in which a portion of their free part must be run within the time window as described in 34. Let $l s t_{i}^{E F}$ be the latest start time of the free part of an activity, i.e., $l c t_{i}-p_{i}^{E F}$. Then activity $i$ 's free part consumes at least $r_{i} \cdot\left(l c t_{b}-l s t_{i}^{E F}\right)$ energy units in $\left[e s t_{a}, l c t_{b}\right)$ if $e^{2} t_{a} \leq e s t_{i}$ and $l s t_{i}^{E F}<l c t_{b}$. We define the energy contributed by such activities by $\operatorname{rs} \operatorname{En}(a, b):=$ $\sum_{i \in \mathcal{V} \backslash \mathcal{V} E F(a, b): e s t_{a} \leq e s t_{i}} \max \left(0, l_{c t}-l s t_{i}^{E F}\right)$.

In summary, TTEF propagation considers three ways in which an activity $i$ can contribute to energy consumption within a time window determined by a task interval $\mathcal{V}^{E F}(a, b)$. First, the free parts that must fully be executed in the time window; second, the compulsory parts that must lies in the time window; and third, some free parts that must partially be run in the time window. Thus, the considered length of an activity $i$ is

$$
p_{i}(a, b):=\left\{\begin{array}{rl}
p_{i} & i \in \mathcal{V}^{E F}(a, b) \\
\max \left(0, l_{c} t_{b}-l s t_{i}\right) & i \notin \mathcal{V}^{E F}(a, b) \wedge \text { est }_{a} \leq \text { est }_{i} \\
\max \left(0, \min \left(l c t_{b}, e c t_{i}\right)-\max \left(e s t_{a}, l s t_{i}\right)\right) & \text { others }
\end{array}\right.
$$

The considered energy consumption is $e_{i}(a, b):=r_{i} \cdot p_{i}(a, b)$ in the time window. 


\subsection{Explanation for the TTEF Consistency Check}

The consistency check is one part of TTEF propagation that checks whether there is a resource overload in any task interval.

Proposition 3.1 (Consistency Check). The cumulative resource scheduling problem is inconsistent if

$$
R \cdot\left(l_{c t}-e s t_{a}\right)-\operatorname{energy}(a, b)<0 \rightarrow \perp
$$

where energy $(a, b):=\sum_{i \in \mathcal{V}^{E F}(a, b)} e_{i}^{E F}+t t E n(a, b)+\operatorname{rsEn}(a, b)$.

This check can be done in $\mathcal{O}\left(l^{2}+n\right)$ runtime, where $l=\left|\mathcal{V}^{E F}\right|$, if the resource profile is given. The corresponding algorithm is shown in Alg. 1 in App. A.

A naïve explanation for a resource overload in the time window $\left[e s t_{a}, l c t_{b}\right)$ only considers the current bounds on activities' start times $S_{i}$.

$$
\bigwedge_{i \in \mathcal{V}: p_{i}(a, b)>0} \llbracket e s t_{i} \leq S_{i} \rrbracket \wedge \llbracket S_{i} \leq l s t_{i} \rrbracket \rightarrow \perp
$$

However, we can easily generalise this explanation by only ensuring that at least $p_{i}(a, b)$ time units are executed in the time window. This results in the following explanation.

$$
\bigwedge_{i \in \mathcal{V}: p_{i}(a, b)>0} \llbracket e s t_{a}+p_{i}(a, b)-p_{i} \leq S_{i} \rrbracket \wedge \llbracket S_{i} \leq l c t_{b}-p_{i}(a, b) \rrbracket \rightarrow \perp
$$

Note that this explanation expresses a resource overload with respect to energetic reasoning propagation which is more general than TTEF.

Let $\Delta:=\operatorname{energy}(a, b)-R \cdot\left(l_{c t}-e s t_{a}\right)-1$. If $\Delta>0$ then the resource overload has extra energy. We can use this extra energy to further generalise the explanation, by reducing the energy required to appear in the time window by up to $\Delta$. For example, if $r_{i} \leq \Delta$ then the lower and upper bound on $S_{i}$ can simultaneously be decreased and increased by a total amount in $\left\{1,2, \ldots, \min \left(\left\lfloor\Delta / r_{i}\right\rfloor, p_{i}(a, b)\right)\right\}$ units without resolving the overload. If $r_{i} \cdot p_{i}(a, b) \leq \Delta$ then we can remove activity $i$ completely from the explanation. In a greedy manner, we try to maximally widen the bounds of activities $i$ where $p_{i}(a, b)>0$, first considering activities with non-empty free parts. If $\Delta_{i}$ denotes the time units of the widening then it holds $p_{i}(a, b) \geq \Delta_{i} \geq 0$ and $\sum_{i \in \mathcal{V}: p_{i}(a, b)>0} \Delta_{i} \cdot r_{i} \leq \Delta$ and we create the following explanation.

$$
\bigwedge_{i \in \mathcal{V}: p_{i}(a, b)-\Delta_{i}>0} \llbracket e s t_{a}+p_{i}(a, b)-p_{i}-\Delta_{i} \leq S_{i} \rrbracket \wedge \llbracket S_{i} \leq l c t_{b}-p_{i}(a, b)+\Delta_{i} \rrbracket \rightarrow \perp
$$

The last generalisation mechanism can be performed in different ways, e.g. we could widen the bounds of activities that were involved in many recent conflicts. Further study is required to identify which are the most appropriate. 


\subsection{Explanation for the TTEF Start Times Propagation}

Propagation on the lower and upper bounds of the start time variables $S_{i}$ are symmetric; Consequently we only present the case for the lower bounds' propagation. To prune the lower bound of an activity $u$, ТTEF bounds propagation tentatively starts the activity $u$ at its earliest start time $e^{s t} t_{u}$ and then checks whether that causes a resource overload in any time window $\left[e^{2} t_{a}, l_{c t}\right)\left(\{a, b\} \subseteq \mathcal{V}^{E F}\right)$. Thus, bounds propagation and its explanation are very similar to that of the consistency check.

The work of [34] considers four positions of $u$ relative to the time window: right (est $t_{a} \leq$ est $\left._{u}<l c t_{b}<e c t_{u}\right)$, inside $\left(\right.$ est $_{a} \leq$ est $\left._{u}<e c t_{u} \leq l c t_{b}\right)$, through $_{\left(e s t_{u}<\text { est }_{a} \wedge \text { lct }_{b}<\right.}$ $\left.e c t_{u}\right)$, and left $\left(e s t_{u}<e s t_{a}<e c t_{u} \leq l c t_{b}\right)$. The first two of these positions correspond to edge-finding propagation and the last two to extended edge-finding propagation. We first consider only the right and inside positions, i.e., est ${ }_{a} \leq e s t_{u}$. Note that $a$ could be $u$. Then,

$$
R \cdot\left(l_{c t}-e s t_{a}\right)-\operatorname{energy}(a, b, u)<0 \rightarrow\left\lceil\frac{\operatorname{rest}(a, b, u)}{r_{u}}\right\rceil \leq S_{u}
$$

where $\operatorname{energy}(a, b, u):=\operatorname{energy}(a, b)-e_{u}(a, b)+r_{u} \cdot\left(\min \left(l_{c t}\right.\right.$, ect $\left.\left._{u}\right)-e \mathrm{st}_{u}\right)$ and

$$
\begin{array}{r}
\operatorname{rest}(a, b, u):=\operatorname{energy}(a, b, u)-\left(R-r_{u}\right) \cdot\left(l c t_{b}-e s t_{a}\right) \\
-r_{u} \cdot\left(\min \left(l_{c t}, e c t_{u}\right)-e s t_{u}\right) .
\end{array}
$$

The first two terms in the sum of energy $(a, b, u)$ gives the energy consumption of all considered activities except $u$, whereas the last term is the required energy of $u$ if it is scheduled at $e s t_{u}$ in the time window $\left[e s t_{a}, l c t_{b}\right)$. The propagation, including explanation generation, can be performed in $\mathcal{O}\left(l^{2}+k \cdot n\right)$ runtime, where $l=\left|\mathcal{V}^{E F}\right|$ and $k$ the number of bounds' updates, if the resource profile is given. Moreover, TTEF propation does not necessarily consider each $u \in \mathcal{V}^{E F}$, but those only that maximise $\min \left(e_{u}^{E F}, r_{u} \cdot\left(l_{c t}-\right.\right.$ $\left.\left.e s t_{a}\right)\right)-r_{u} \cdot \max \left(0, l c t_{b}-l s t_{u}^{E F}\right)$ and satisfy $e s t_{a} \leq e s t_{u}$. The corresponding algorithm is shown in Alg. 2 in App. A.

A naïve explanation for a lower bound update from est ${ }_{u}$ to new $L B:=\left\lceil\operatorname{rest}(a, b, u) / r_{u}\right\rceil$ with respect to the time window $\left[e s t_{a}, l c t_{b}\right)$ additionally includes the previous and new lower bound on the left and right hand side of the implication, respectively, in comparison to the naïve explanation for a resource overload.

$$
\llbracket e s t_{u} \leq S_{u} \rrbracket \wedge \bigwedge_{i \in \mathcal{V} \backslash\{u\}: p_{i}(a, b)>0} \llbracket e s t_{i} \leq S_{i} \rrbracket \wedge \llbracket S_{i} \leq l s t_{i} \rrbracket \rightarrow \llbracket n e w L B \leq S_{u} \rrbracket
$$

As we discussed in the case of resource overload, we perform a similar generalisation for the activities in $\mathcal{V} \backslash\{u\}$, and for $u$ we decrease the lower bound on the left hand side as much as possible so that the same propagation holds when $u$ is executed at that 
Table 1: Specifications of the benchmark suites.

\begin{tabular}{|c|c|c|c|c|c|c|}
\hline suite & sub-suites & \#inst & \#act & $p_{i}$ & \#res & notes \\
\hline AT [3] & ST27/sT51/sT103 & 48 each & $25 / 49 / 101$ & $1-12$ & 6 each & \\
\hline PSPLib [16] & J30 [17]/J60/J90 & 480 each & $30 / 60 / 90$ & $1-10$ & 4 each & \\
\hline BL 4] & BL20/BL25 & 20 each & $20 / 25$ & $\frac{1-10}{1-6}$ & 3 each & \\
\hline PACK [ 8$]$ & & 55 & $15-33$ & $1-19$ & $2-5$ & \\
\hline KSD15_D [18 & & 480 & 15 & $1-250$ & 4 & based on J30 \\
\hline PACK_D [18] & & 55 & $15-33$ & $1-1138$ & $2-5$ & $\begin{array}{ll}\text { based } & \text { on } \\
\text { PACK } & \end{array}$ \\
\hline
\end{tabular}

decreased lower bound.

$$
\begin{aligned}
& \llbracket e s t_{a}+l c t_{b}-n e w L B+1-p_{u} \leq S_{u} \rrbracket \wedge \\
& \bigwedge_{i \in \mathcal{V} \backslash\{u\}: p_{i}(a, b)>0} \llbracket e s t_{a}+p_{i}(a, b)-p_{i} \leq S_{i} \rrbracket \wedge \llbracket S_{i} \leq l c t_{b}-p_{i}(a, b) \rrbracket \\
& \quad \rightarrow \llbracket n e w L B \leq S_{u} \rrbracket
\end{aligned}
$$

Again this more general explanation expresses the energetic reasoning propagation and the bounds of activities in $\left\{i \in \mathcal{V} \backslash\{u\} \mid p_{i}(a, b)>0\right\}$ can further be generalised in the same way as for a resource overload. But here the available energy units $\Delta$ for widening the bounds is $\operatorname{rest}(a, b, u)-r_{u} \cdot($ newL $B-1)+1$. Hence, $0 \leq \Delta<r_{u}$ indicate that the explanation only can further be generalised a little bit. We perform this generalisation as for the overload case.

\section{Experiments on Resource-constrained Project Scheduling Problems}

We carried out extensive experiments on RCPSP instances comparing our solution approach using both time-table and/or TTEF propagation. We compare the obtained results on the lower bounds of the makespan with the best known so far. Detailed results are available at http://www.cs.mu.oz.au/ pjs/rcpsp.

We used six benchmark suites for which an overview is given in Tab. 1 where \#inst, \#act, $p_{i}$, and \#res are the number of instances, number of activities, range of processing times, and number of resources, respectively. The first two suites are highly disjunctive, while the remainder are highly cumulative.

The experiments were run on a X86-64 architecture running GNU/Linux and a Intel(R) Core(TM) i7 CPU processor at 2.8GHz. The code was written in Mercury [30] using the G12 Constraint Programming Platform [31].

We model an instance as in [27] using global cumulative constraints cumulative and difference logic constraints $\left(S_{i}+p_{i} \leq S_{j}\right)$, resp. In addition, between two activities $i, j$ in disjunction, i.e., two activities which cannot concurrently run without overloading some 
resource, the two half-reified constraints [10] $b \rightarrow S_{i}+p_{i} \leq S_{j}$ and $\neg b \rightarrow S_{j}+p_{j} \leq S_{i}$ are posted where $b$ is a Boolean variable.

We run cumulative constraint propagation using different phases:

(a) time-table consistency check in $\mathcal{O}(n+p \log p)$ runtime,

(b) TTEF consistency check in $\mathcal{O}\left(l^{2}+n\right)$ runtime as defined in Section 3.1 ,

(c) time-table bounds' propagation in $\mathcal{O}(l \cdot p+k \cdot \min (R, n))$ runtime, and

(d) TTEF bounds' propagation in $\mathcal{O}\left(l^{2}+k \cdot n\right)$ runtime as defined in Section 3.2 where $k, l, n, p$ are the numbers of bounds' updates, unfixed activities, all activities, and height changes in the resource profile, resp.

Note that in our setup phase (d) TTEF bounds' propagation does not take into account the bounds' changes of the phase (c) time-table bounds' propagation. For the experiments, we consider three settings of the cumulative propagator: tt executes phases (a) and $(\mathrm{c})$, ttef(c) $(\mathrm{a}-\mathrm{c})$, and ttef $(\mathrm{a}-\mathrm{d})$. Note that phases $(\mathrm{c})$ and $(\mathrm{d})$ are not run if either phase (a) or (b) detects inconsistency.

\subsection{Upper Bound Computation}

For solving RCPSP we use the same branch-and-bound algorithm as we used in [27, but here we limit ourselves to the search heuristic HotRESTART which was the most robust one in our previous studies [26, 27]. It executes an adapted search of [4] using serial scheduling generation for the first 500 choice points and, then, continues with an activity based search (a variant of VsIDS [20]) on the Boolean variables representing a lower part $x \leq v$ and upper part $v<x$ of the variable $x$ 's domain where $x$ is either a start time or the makespan variable and $v$ a value of $x$ 's initial domain. Moreover, it is interleaved with a geometric restart policy [35] on the number of node failures for which the restart base and factor are 250 failures and 2.0, respectively. The search was halted after 10 minutes.

The results are given in Tab. 2 and 3 . For each benchmark suite, the number of solved instances (\#svd) is given. The column $\operatorname{cmpr}(a)$ shows the results on the instances solved by all methods, where $a$ is the number of such instances. The left entry in that column is the average runtime on these instances in seconds, and the right entry is the average number of failures during search. The entries in column all $(a)$ have the same meaning, but here all instances are considered where $a$ is the total number of instances. For unsolved instances, the number of failures after 10 minutes is used.

Table 2 shows the results on the highly disjunctive RCPsPs. As expected, the stronger propagation (ttef(c), ttef) reduces the search space overall in comparision to tt, but the average runtime is higher by a factor of about $5 \%-70 \%$ and $50 \%-100 \%$ for ttef(c) and ttef. Interestingly, ttef(c) and ttef solved respectively 1 and 2 more instances on J60 and closed the instance j120_1_1 on J120 which has an optimal makespan 105. This makespan corresponds to the best known upper bound. However, the stronger propagation does not generally pay off for a CP solver with nogood learning. 
Table 2: UB results on highly disjunctive RCPsPs.

\begin{tabular}{|c|c|c|c|c|c|c|c|c|c|c|}
\hline \multirow[b]{3}{*}{$t$} & \multicolumn{5}{|c|}{$\mathrm{J} 30$} & \multicolumn{5}{|c|}{ J60 } \\
\hline & \#svd & $\mathrm{cmp}$ & $(480)$ & \multicolumn{2}{|c|}{ all $(480)$} & \#svd & $\mathrm{cmpr}$ & (429) & \multicolumn{2}{|c|}{ all(480) } \\
\hline & 480 & 0.12 & 1074 & 0.12 & 1074 & 430 & 1.82 & 5798 & 64.25 & 93164 \\
\hline ttef(c) & 480 & 0.20 & 1103 & 0.20 & 1103 & 431 & 2.00 & 4860 & 64.39 & 80845 \\
\hline \multirow[t]{3}{*}{ ttef } & 480 & 0.23 & 991 & 0.23 & 991 & 432 & 3.04 & 5191 & 64.87 & 62534 \\
\hline & \multicolumn{5}{|c|}{ J90 } & \multicolumn{5}{|c|}{$\mathrm{J} 120$} \\
\hline & \#svd & \multicolumn{2}{|c|}{$\operatorname{cmpr}(400)$} & \multicolumn{2}{|c|}{$\operatorname{all}(480)$} & \#svd & \multicolumn{2}{|c|}{$\operatorname{cmpr}(280)$} & \multicolumn{2}{|c|}{$\operatorname{all}(600)$} \\
\hline $\mathrm{tt}$ & 400 & 5.03 & 9229 & 104.09 & 132234 & 283 & 9.71 & 15022 & 322.35 & 398941 \\
\hline ttef(c) & 400 & 6.93 & 9512 & 105.69 & 104297 & 282 & 13.47 & 16958 & 324.73 & 297562 \\
\hline \multirow[t]{3}{*}{ ttef } & 400 & 8.10 & 8830 & 106.66 & 72402 & 283 & 14.97 & 13490 & 324.66 & 186597 \\
\hline & \multicolumn{5}{|c|}{$\overline{\mathrm{AT}}$} & & & & & \\
\hline & \#svd & $\mathrm{cmp}$ & (129) & \multicolumn{2}{|c|}{ all(144) } & & & & & \\
\hline tt & 132 & 8.90 & 19997 & 66.22 & 87226 & & & & & \\
\hline ttef(c) & 130 & 9.36 & 16466 & 69.41 & 72056 & & & & & \\
\hline ttef & 129 & 13.55 & 17239 & 74.60 & 63554 & & & & & \\
\hline
\end{tabular}

Table 3: UB results on highly cumulative RCPSPs.

\begin{tabular}{|c|c|c|c|c|c|c|c|c|c|}
\hline & \multicolumn{5}{|c|}{$\mathrm{BL}$} & \multicolumn{4}{|c|}{ PACK } \\
\hline & \#svd & $\mathrm{cmpl}$ & $r(40)$ & \multicolumn{2}{|c|}{$\operatorname{all}(40)$} & \#svd & $\mathrm{cml}_{\mathrm{l}}$ & $\operatorname{pr}(16)$ & $\operatorname{all}(55)$ \\
\hline \multirow{5}{*}{$\begin{array}{l}\text { tt } \\
\text { ttef(c) } \\
\text { ttef }\end{array}$} & 40 & 0.16 & 2568 & 0.16 & 2568 & 16 & 77.65 & 245441 & $\begin{array}{|ll|}447.69 & 699615\end{array}$ \\
\hline & 40 & 0.02 & 370 & 0.02 & 370 & 39 & 37.22 & 122038 & $\begin{array}{lll}186.79 & 292101\end{array}$ \\
\hline & 40 & 0.02 & 269 & 0.02 & 269 & 39 & 44.44 & 105751 & $188.23 \quad 257747$ \\
\hline & \multicolumn{5}{|c|}{ KSSD15_D } & \multicolumn{4}{|c|}{$\begin{array}{l}\text { PACK_D } \\
\end{array}$} \\
\hline & \#svd & \multicolumn{2}{|c|}{ cmpr(480) } & \multicolumn{2}{|c|}{ all(480) } & $\#$ svd & \multicolumn{2}{|c|}{$\operatorname{cmpr}(37)$} & all(55) \\
\hline & 480 & 0.01 & 26 & 0.01 & 26 & 37 & 32.72 & 42503 & $218.26 \quad 184293$ \\
\hline & 480 & 0.01 & 26 & 0.01 & 26 & 37 & 23.96 & 32916 & 212.37170301 \\
\hline ttef & 480 & 0.01 & 26 & 0.01 & 26 & 37 & 36.93 & 37004 & $221.11 \quad 157015$ \\
\hline
\end{tabular}


Table 4: LB results on AT, PACK, and PACK_D

\begin{tabular}{|c|c|c|c|}
\hline & AT & PACK & PACK_D \\
\hline ttef(c & $5 / 4 / 3+52$ & $0 / 4 / 12+100$ & $0 / 7 / 11+632$ \\
\hline & $7 / 2 / 3+44$ & $1 / 4 / 11+101$ & $2 / 5 / 10+618$ \\
\hline
\end{tabular}

Table 5: LB results on J60, J90, and J120

\begin{tabular}{|c|c|c|c|c|c|c|c|c|c|c|c|c|c|c|c|c|c|c|c|}
\hline & \multicolumn{3}{|c|}{ J60 } & \multicolumn{5}{|c|}{ J90 } & \multicolumn{10}{|c|}{ J120 } \\
\hline & & +1 & +2 & +3 & +1 & +2 & +3 & +4 & +5 & +1 & +2 & +3 & +4 & +5 & +6 & +7 & +8 & +9 & +10 \\
\hline \multirow{2}{*}{$1 \mathrm{~min}$} & ttef(c) & 4 & 1 & - & 12 & 1 & - & - & - & 27 & 8 & 4 & - & - & - & 2 & - & - & - \\
\hline & ttef & 7 & 5 & - & 25 & 14 & 3 & 1 & - & 90 & 20 & 10 & 5 & 2 & - & - & 2 & - & - \\
\hline \multirow{2}{*}{$10 \mathrm{mins}$} & ttef(c) & 21 & 2 & - & 25 & 7 & - & - & - & 68 & 16 & 4 & 4 & 2 & - & - & 1 & 1 & - \\
\hline & & 13 & 6 & 3 & 35 & 17 & 6 & 3 & 1 & 116 & 39 & 9 & 9 & 4 & 1 & - & - & 1 & 1 \\
\hline
\end{tabular}

Table 3 presents the results on highly cumulative RCPSPs which clearly shows the benefit of TTEF propagation, especially on BL for which ttef(c) and ttef reduce the search space and the average runtime by a factor of 8 , and PACK for which they solved 23 instances more than tt. On PACK_D, ttef(c) is about $50 \%$ faster on average than tt while ttef is slightly slower on average than tt. No conclusion can be drawn on KSD15_D because the instances are easy for LCG solvers.

\subsection{Lower Bound Computation}

The lower bound computation tries to solve RCPSPs in a destructive way by converging to the optimal makespan from below, i.e., it repeatedly proves that there exists no solution for current makespan considered and continues with an incremented makespan by 1 . If a solution found then it is the optimal one. For these experiments we use the search heuristic HotStaRT as we did in [26, 27]. This heuristic is HotRestart (as decribed earlier) but no restart. We used the same parameters as for HотRESTART. For the starting makespan, we choose the best known lower bounds on J60, J90, and J120 recorded in the PSPLib at http://129.187.106.231/psplib/and [34 at http:// vilim.eu/petr/cpaior2011-results.txt. On the other suites, the search starts from makespan 1. Due to the tighter makespan, it is expected that the TTEF propagation will perform better than for upper bound computation on the highly disjunctive instances. The search was cut off at 10 minutes as in [26, 27].

Table 4 shows the results on AT, PACK, and PACK_D restricted to the instances that none of the methods could solve using the upper bound computation, that are 12, 16, and 18 for AT, PACK, and PACK_D, respectively. An entry $a / b / c$ for method $x$ means that $x$ achieved respectively $a$-times, $b$-times and $c$-times a worse, the same and a better lower bound than tt. The entry $+d$ is the sum of lower bounds' differences of method $x$ to tt. On PACK and PACK_D, ttef(c) and ttef clearly perform better than tt. On the highly disjunctive instances in $\mathrm{AT}$, ttef(c) and tt are almost balanced whereas tt could generate better lower bounds on more instances as ttef. The lower bounds' differences on AT are dominated by the instance st103_4 for which ttef(c) and ttef retrieved a lower 
bound improvement of 54 and 53 time periods with respect to tt.

The more interesting results are presented in Tab. 5 because the best lower bounds are known for all the remaining open instances $\left(48,77,307\right.$ in J60, J90, J120) ${ }^{2}$ An entry in a column $+d$ shows the number of instances for that the corresponding method could improve the lower bound by $d$ time periods. On these instances, we run at first the experiments with a runtime limit of one minute as it was done in the experiments for TTEF propagation in 34 but he used a CP solver without nogood learning. tt could not improve any lower bound because its corresponding results are already recorded in the PSPLib. ttef(c) and ttef improved the lower bounds of 59 and 183 instances, respectively, which is about $13.7 \%$ and $42.4 \%$ of the open instances. Although, the experiments in 34] were run on a slower machine ${ }^{3}$ the results confirm the importance of nogood learning. For the experiments with 10 minutes runtime, we excluded tt due to time constraints and expected inferior results to ttef(c) and ttef. With the extended runtime, ttef(c) and ttef could improved the lower bounds of more instance, namely 151 and 264 instances, respectively, which is about $35.0 \%$ and $61.1 \%$. Moreover, 3,1 , and 1 of the remaining open instances on J60, J90, and J120, respectively, could be solved optimally. See App. B for the listing of the closed instances and the new lower bounds.

\section{Conclusion and Outlook}

We present explanations for the recently developed TTEF propagation of the global cumulative constraint for lazy clause generation solvers. These explanations express an energetic reasoning propagation which is a stronger propagation than the TTEF one.

Our implementation of this propagator was compared to time-table propagation in lazy clause generation solvers on six benchmark suites. The preliminary results confirms the importance of energy-based reasoning on highly disjunctive RCPSPs for CP solvers with nogood learning.

Moreover, our approach with TTEF propagation was able to close one instance. It also improves the best known lower bounds for 264 of the remaining 432 remaining open instances on RCPSPs from the PSPLib.

In the future, we want to integrate the extended edge-finding propagation into TTEF propagation as it was originally proposed in [34], to perform experiments on cutting and packing problems, and to study different variations of explanations for TTEF propagation. Furthermore, we want to look at a more efficient implementation of the TTEF propagation as well as an implementation of energetic reasoning.

Acknowledgements NICTA is funded by the Australian Government as represented by the Department of Broadband, Communications and the Digital Economy and the Australian Research Council through the ICT Centre of Excellence program. This work

\footnotetext{
${ }^{2}$ Note that the PSPLib still lists the instances j60_25_5, j90_26_5, j120_8_3, j120_48_5, and j120_35_5 as open, but we closed the first four ones in [27] and [19] closed the last one.

${ }^{3} \operatorname{Intel}(\mathrm{R})$ Core(TM)2 Duo CPU T9400 on $2.53 \mathrm{GHz}$
} 
was partially supported by Asian Office of Aerospace Research and Development grant $10-4123$.

\section{References}

[1] Tobias Achterberg. SCIP: solving constraint integer programs. Mathematical Programming Computation, 1:1-41, 2009. ISSN 1867-2949. doi: 10.1007/ s12532-008-0001-1.

[2] Abderrahmane Aggoun and Nicolas Beldiceanu. Extending CHIP in order to solve complex scheduling and placement problems. Mathematical and Computer Modelling, 17(7):57-73, 1993.

[3] Ramón Alvarez-Valdés and José Manuel Tamarit. Advances in Project Scheduling, chapter Heuristic algorithms for resource-constrained project scheduling: A review and an empirical analysis, pages 113-134. Elsevier, 1989.

[4] Philippe Baptiste and Claude Le Pape. Constraint propagation and decomposition techniques for highly disjunctive and highly cumulative project scheduling problems. Constraints, 5(1-2):119-139, 2000.

[5] Philippe Baptiste, Claude Le Pape, and Wim Nuijten. Satisfiability tests and timebound adjustments for cumulative scheduling problems. Annals of Operations Research, 92:305-333, 1999. doi: 10.1023/A:1018995000688.

[6] Philippe Baptiste, Claude Le Pape, and Wim Nuijten. Constraint-Based Scheduling. Kluwer Academic Publishers, Norwell, MA, USA, 2001. ISBN 0792374088.

[7] Timo Berthold, Stefan Heinz, Marco Lübbecke, Rolf Möhring, and Jens Schulz. A constraint integer programming approach for resource-constrained project scheduling. In Andrea Lodi, Michela Milano, and Paolo Toth, editors, Integration of AI and OR Techniques in Constraint Programming for Combinatorial Optimization Problems, volume 6140 of Lecture Notes in Computer Science, pages 313-317. Springer Berlin / Heidelberg, 2010. doi: 10.1007/978-3-642-13520-0_34.

[8] Jacques Carlier and Emmanuel Néron. On linear lower bounds for the resource constrained project scheduling problem. European Journal of Operational Research, 149(2):314-324, 2003. ISSN 0377-2217. doi: 10.1016/S0377-2217(02)00763-4.

[9] Thibaut Feydy and Peter J. Stuckey. Lazy clause generation reengineered. In Gent [11], pages 352-366. doi: 10.1007/978-3-642-04244-7_29.

[10] Thibaut Feydy, Zoltan Somogyi, and Peter J. Stuckey. Half reification and flattening. In Jimmy Ho-Man Lee, editor, Proceedings of Principles and Practice of Constraint Programming - CP 2011, volume 6876 of Lecture Notes in Computer Science, pages 286-301. Springer, 2011. 
[11] Ian P. Gent, editor. Proceedings of Principles and Practice of Constraint Programming - CP 2009, volume 5732 of Lecture Notes in Computer Science, 2009. Springer Berlin / Heidelberg.

[12] Stefan Heinz and Jens Schulz. Explanations for the cumulative constraint: An experimental study. In Panos M. Pardalos and Steffen Rebennack, editors, Proceedings of Experimental Algorithms - SEA 2011, volume 6630 of Lecture Notes in Computer Science, pages 400-409. Springer Berlin / Heidelberg, 2011. doi: 10.1007/978-3-642-20662-7_34.

[13] Narendra Jussien. The versatility of using explanations within constraint programming. Research Report 03-04-INFO, École des Mines de Nantes, Nantes, France, 2003.

[14] Narendra Jussien and Vincent Barichard. The PaLM system: explanation-based constraint programming. In Proceedings of TRICS: Techniques foR Implementing Constraint programming Systems, a post-conference workshop of CP 2000, pages 118-133, Singapore, 2000.

[15] George Katsirelos and Fahiem Bacchus. Generalized nogoods in CSPs. In Manuela M. Veloso and Subbarao Kambhampati, editors, Proceedings on Artificial Intelligence - AAAI 2005, pages 390-396. AAAI Press / The MIT Press, 2005.

[16] Rainer Kolisch and Arno Sprecher. PSPLIB - A project scheduling problem library. European Journal of Operational Research, 96(1):205-216, 1997. doi: 10.1016/ S0377-2217(96)00170-1.

[17] Rainer Kolisch, Arno Sprecher, and Andreas Drexl. Characterization and generation of a general class of resource-constrained project scheduling problems. Management Science, 41(10):1693-1703, 1995. ISSN 0025-1909.

[18] Oumar Koné, Christian Artigues, Pierre Lopez, and Marcel Mongeau. Event-based milp models for resource-constrained project scheduling problems. Computers 83 Operations Research, 38(1):3-13, 2011. doi: 10.1016/j.cor.2009.12.011.

[19] Olivier Liess and Philippe Michelon. A constraint programming approach for the resource-constrained project scheduling problem. Annals of Operations Research, 157(1):25-36, January 2008. doi: 10.1007/s10479-007-0188-y.

[20] Matthew W. Moskewicz, Conor F. Madigan, Ying Zhao, Lintao Zhang, and Sharad Malik. Chaff: Engineering an efficient SAT solver. In Proceedings of Design Automation Conference - DAC 2001, pages 530-535, New York, NY, USA, 2001. ACM. doi: $10.1145 / 378239.379017$.

[21] Wilhelmus Petronella Maria Nuijten. Time and Resource Constrained Scheduling. PhD thesis, Eindhoven University of Technology, 1994. 
[22] Olga Ohrimenko, Peter J. Stuckey, and Michael Codish. Propagation via lazy clause generation. Constraints, 14(3):357-391, 2009.

[23] Christian Schulte and Peter J. Stuckey. Efficient constraint propagation engines. ACM Transactions on Programming Languages and Systems, 31(1):Article No. 2, 2008 .

[24] Andreas Schutt. Improving Scheduling by Learning. PhD thesis, The University of Melbourne, 2011. URL http://repository.unimelb.edu.au/10187/11060.

[25] Andreas Schutt and Armin Wolf. A new $\mathcal{O}\left(n^{2} \log n\right)$ not-first/not-last pruning algorithm for cumulative resource constraints. In David Cohen, editor, Proceedings of Principles and Practice of Constraint Programming - CP 2010, volume 6308 of Lecture Notes in Computer Science, pages 445-459. Springer Berlin / Heidelberg, 2010. URL 10.1007/978-3-642-15396-9_36.

[26] Andreas Schutt, Thibaut Feydy, Peter J. Stuckey, and Mark G. Wallace. Why cumulative decomposition is not as bad as it sounds. In Gent [11], pages 746-761. doi: 10.1007/978-3-642-04244-7_58.

[27] Andreas Schutt, Thibaut Feydy, Peter J. Stuckey, and Mark G. Wallace. Explaining the cumulative propagator. Constraints, 16(3):250-282, 2011. doi: 10.1007/s10601-010-9103-2.

[28] Andreas Schutt, Peter Stuckey, and Andrew Verden. Optimal carpet cutting. In Jimmy Lee, editor, Principles and Practice of Constraint Programming - CP 2011, volume 6876 of Lecture Notes in Computer Science, pages 69-84. Springer Berlin / Heidelberg, 2011. doi: 10.1007/978-3-642-23786-7_8.

[29] Andreas Schutt, Thibaut Feydy, Peter J. Stuckey, and Mark G. Wallace. Solving RCPSP/max by lazy clause generation. Journal of Scheduling, pages 1-18, 2012. doi: $10.1007 / \mathrm{s} 10951-012-0285-\mathrm{x}$.

[30] Zoltan Somogyi, Fergus Henderson, and Thomas Conway. The execution algorithm of Mercury, an efficient purely declarative logic programming language. The Journal of Logic Programming, 29(1-3):17-64, 1996. ISSN 0743-1066. doi: 10.1016/S0743-1066(96)00068-4.

[31] Peter J. Stuckey, Maria J. García de la Banda, Michael J. Maher, Kim Marriott, John K. Slaney, Zoltan Somogyi, Mark G. Wallace, and Toby Walsh. The G12 project: Mapping solver independent models to efficient solutions. In Maurizio Gabbrielli and Gopal Gupta, editors, Proceedings of Logic Programming - ICLP 2005, volume 3668 of Lecture Notes in Computer Science, pages 9-13. Springer Berlin / Heidelberg, October 2005. doi: 10.1007/11562931_3.

[32] Petr Vilím. Computing explanations for the unary resource constraint. In Roman Barták and Michela Milano, editors, Proceedings of Integration of AI and OR 
Techniques in Constraint Programming for Combinatorial Optimization Problems CPAIOR 2005, volume 3524 of Lecture Notes in Computer Science, pages 396-409. Springer Berlin / Heidelberg, 2005. doi: 10.1007/11493853_29.

[33] Petr Vilím. Edge finding filtering algorithm for discrete cumulative resources in $\mathcal{O}(k n \log n)$. In Gent [11, pages 802-816. doi: 10.1007/978-3-642-04244-7_62.

[34] Petr Vilím. Timetable edge finding filtering algorithm for discrete cumulative resources. In Tobias Achterberg and J. Beck, editors, Proceedings of Integration of $A I$ and OR Techniques in Constraint Programming for Combinatorial Optimization Problems - CPAIOR 2011, volume 6697 of Lecture Notes in Computer Science, pages 230-245. Springer Berlin / Heidelberg, 2011. doi: 10.1007/ 978-3-642-21311-3_22.

[35] Toby Walsh. Search in a small world. In Proceedings of Artificial intelligence IJCAI 1999, pages 1172-1177. Morgan Kaufmann, 1999. 


\section{A. TTEF propagation algorithms}

Algorithm 1 shows the TTEF consistency check used. The outer loop (lines 2-16) iterates over all distinctive possible end times for the time windows while the inner loop (lines 7-16) iterates over all possible start times. In line 11 (12), it checks whether a must fully (partially) be executed in the current time window and further ones checked in the same inner loop. If so it adds the required free energy units $e_{a}^{E F}$ of $a$ to $E$. In line 13, it calculates the still available energy units in the time window [begin, end) taking the energy units from the resource profile $\operatorname{tt} \operatorname{En}(a, b)$ into account. If this results in a resource overload then a corresponding explanation is generated (line 15) and the algorithm fails; otherwise, the algorithm succeeds.

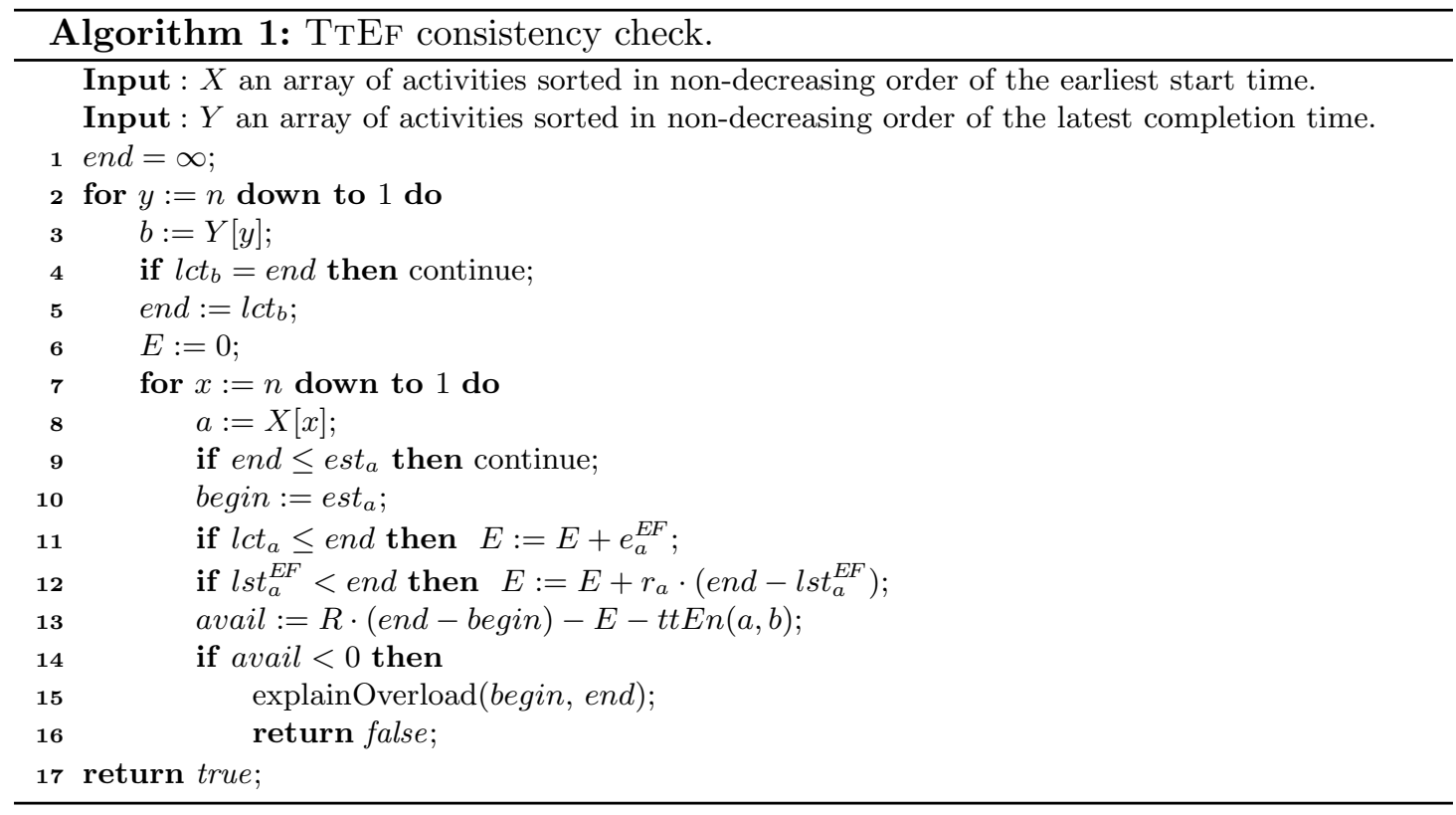

Algorithm 2 shows the lower bounds propagation algorithm. As for Alg. 1 the outer loop (lines 3-24) and inner loop (lines 7-24) iterate over the end and start times of the time windows [begin, end), but require more book keeping. In line 6 , it initialises $E$. $u$, and $e n R e q U$ where: $E$ records the required energy units by the considered activities that must fully or partially be run in the time window; and $u$ stores the activity that maximises $\min \left(e_{u}^{E F}, r_{u} \cdot(\right.$ end - begin $\left.)\right)-r_{u} \cdot \max \left(0\right.$, end $\left.-l s t_{u}^{E F}\right)$ and that value is saved in enReqU. If $a$ must be fully or partially be executed in the time window then the corresponding energy units are added to $E$ in lines 11 and 14, resp. The desired activity for pruning is computed in lines 13,15 , and 16, whereas the available energy units are calculated in line 17. In the case that there is not sufficient energy available then the condition of line 18 holds and the algorithm determines the first possible start time for $u$ (lines 19, 20). If that is larger than the recorded earliest start time in est $t_{u}^{\prime}$ then the algorithm generates the explanation (line 22) and postpones the update (line 23) after finishing with the outer loop (line 25). 


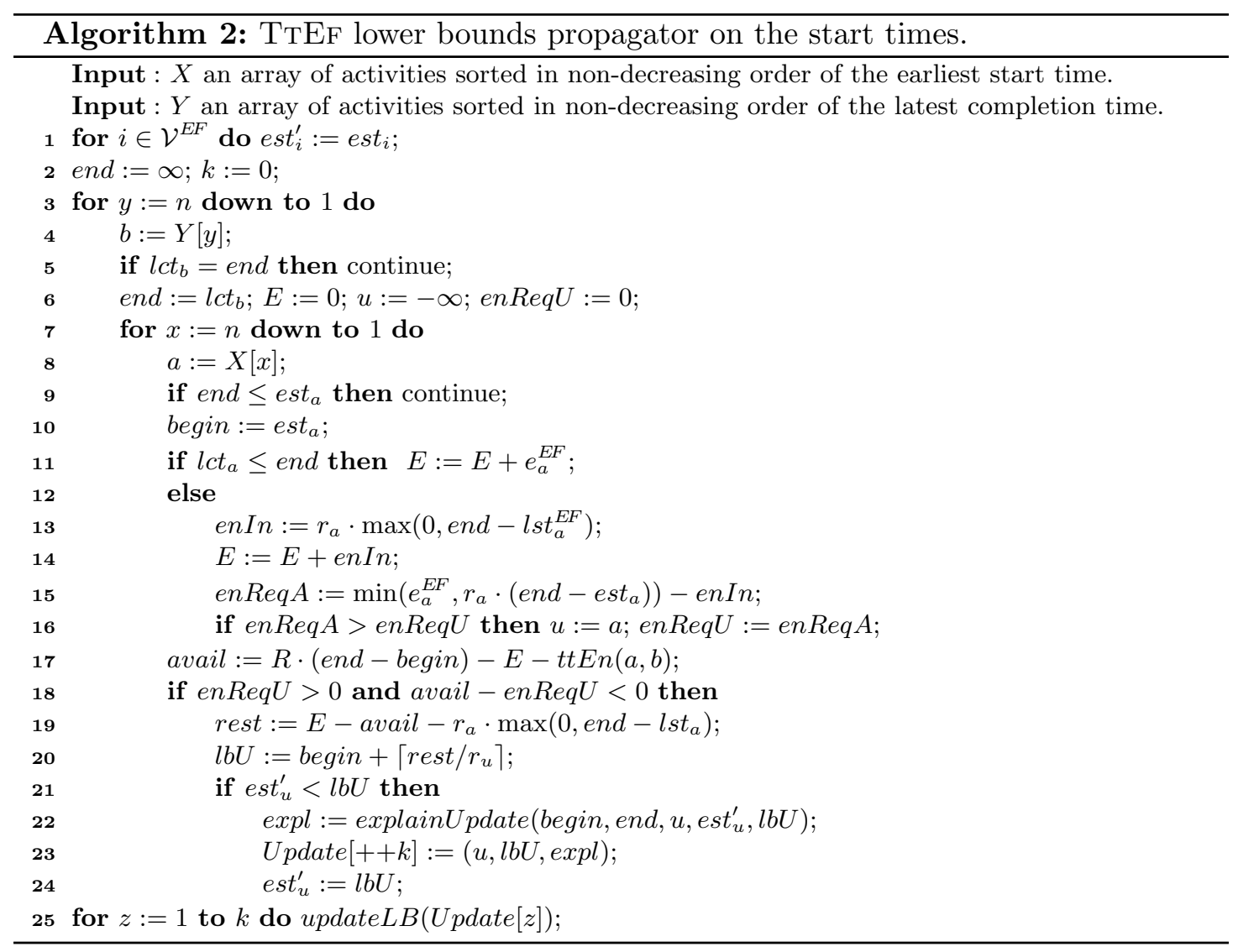


Table 6: New lower bounds on J60.

\begin{tabular}{cc|cc|cc|cc|cc|cc|cc} 
inst & LB & inst & LB & inst & LB & inst & LB & inst & LB & inst & LB & inst & LB \\
\hline $9 \_1$ & 85 & $9 \_5$ & 81 & $9 \_6$ & 106 & $9 \_7$ & 103 & $9 \_8$ & 95 & $9 \_10$ & 89 & $13 \_1$ & 105 \\
$13 \_2$ & 103 & $13 \_3$ & 84 & $13 \_4$ & 98 & $13 \_7$ & 82 & $13 \_8$ & 115 & $13 \_9$ & 96 & $13 \_10$ & 113 \\
$25 \_2$ & 96 & $25 \_4$ & 106 & $25 \_6$ & 106 & $29 \_1$ & 97 & $29 \_6$ & 144 & $29 \_7$ & 115 & $29 \_8$ & 97 \\
$41 \_3$ & 90 & $41 \_10$ & 106 & $45 \_1$ & 90 & & & & & & & &
\end{tabular}

Table 7: New lower bounds on J90.

\begin{tabular}{cc|cc|cc|cc|cc|cc|cc} 
inst & LB & inst & LB & inst & LB & inst & LB & inst & LB & inst & LB & inst & LB \\
\hline $5 \_3$ & 84 & $5 \_5$ & 109 & $5 \_7$ & 106 & $5 \_8$ & 97 & $5 \_9$ & 114 & $5 \_10$ & 95 & $9 \_2$ & 122 \\
$9 \_3$ & 98 & $9 \_4$ & 120 & $9 \_5$ & 127 & $9 \_6$ & 113 & $9 \_7$ & 103 & $9 \_8$ & 111 & $9 \_9$ & 106 \\
$9 \_10$ & 105 & $13 \_2$ & 119 & $13 \_3$ & 105 & $13 \_5$ & 109 & $13 \_7$ & 116 & $13 \_8$ & 113 & $13 \_9$ & 117 \\
$13 \_10$ & 114 & $21 \_7$ & 106 & $21 \_8$ & 108 & $25 \_1$ & 117 & $25 \_2$ & 122 & $25 \_3$ & 113 & $25 \_4$ & 128 \\
$25 \_5$ & 110 & $25 \_6$ & 113 & $25 \_8$ & 131 & $25 \_9$ & 98 & $25 \_10$ & 119 & $29 \_1$ & 126 & $29 \_2$ & 122 \\
$29 \_4$ & 139 & $29 \_6$ & 117 & $29 \_7$ & 160 & $29 \_8$ & 146 & $29 \_9$ & 120 & $30 \_9$ & 92 & $37 \_2$ & 114 \\
$41 \_1$ & 129 & $41 \_2$ & 154 & $41 \_3$ & 149 & $41 \_4$ & 142 & $41 \_5$ & 116 & $41 \_6$ & 124 & $41 \_7$ & 145 \\
$41 \_8$ & 148 & $41 \_9$ & 110 & $41 \_10$ & 144 & $45 \_1$ & 143 & $45 \_2$ & 138 & $45 \_3$ & 144 & $45 \_4$ & 126 \\
$45 \_6$ & 163 & $45 \_7$ & 129 & $45 \_8$ & 150 & $45 \_9$ & 145 & $45 \_10$ & 156 & $46 \_9$ & 86 & &
\end{tabular}

\section{B. Closed Instances and New Lower Bounds on PSPLib}

From the open instances, we closed the instances 9_3 (100), 9_9 (99), 25_10 (108) on J60, $5 \_6(86)$ on J90, and 1_1 (105), 8_6 (85) on J120 where the number in brackets shows the optimal makespan. We computed new lower bounds on the remaining open instances from the PSPLib. Tables 68 list these new lower bounds where the column "inst" shows the name of the instance and the column "LB" the corresponding new lower bound.

\section{Best Lower and Upper Bounds Retrieved}

For a later comparison, Tables 911 show the best lower and upper bounds for AT, PACK, and PACK_D retrieved by one of the methods tt, ttef(c), and ttef. The column "inst" shows the instance name and the column "LB/UB" the corresponding lower and upper bound. If these bounds are equal then only one number is given. 
Table 8: New lower bounds on J120.

\begin{tabular}{|c|c|c|c|c|c|c|c|c|c|c|c|c|c|}
\hline inst & LB & inst & LB & inst & LB & inst & LB & inst & LB & inst & LB & inst & LB \\
\hline $6 \_1$ & 134 & $6 \_2$ & 127 & $6 \_5$ & 117 & 6_6 & 141 & $6 \_8$ & 141 & $6 \_9$ & 150 & 6_10 & 158 \\
\hline 7_1 & 99 & 7_3 & 98 & 7_4 & 106 & 7_6 & 116 & 7_7 & 114 & 7_8 & 93 & 7_9 & 87 \\
\hline 7_10 & 112 & 8_2 & 102 & 8_5 & 100 & 8_9 & 90 & 8_10 & 92 & 9_4 & 85 & 11_1 & 157 \\
\hline 11_2 & 147 & 11_3 & 189 & 11_4 & 178 & 11_5 & 194 & 11_6 & 192 & 11_7 & 149 & 11_8 & 153 \\
\hline 11_10 & 164 & 12_1 & 126 & 12_2 & 112 & 12_4 & 122 & $12 \_5$ & 155 & 12_6 & 116 & 13_1 & 124 \\
\hline 13_3 & 116 & 13_4 & 109 & 13_6 & 96 & 13_9 & 83 & 14_2 & 91 & $14 \_5$ & 94 & $14 \_7$ & 90 \\
\hline 16_1 & 181 & 16_3 & 221 & 16_4 & 191 & 16_6 & 195 & 16_8 & 183 & $17 \_5$ & 124 & $17 \_6$ & 134 \\
\hline 18_8 & 102 & 18_9 & 89 & 18_10 & 97 & 26_1 & 155 & 26_2 & 159 & 26_3 & 158 & 26_4 & 161 \\
\hline 26_5 & 139 & 26_6 & 171 & $26 \_7$ & 147 & 26_8 & 168 & 26_9 & 161 & 26_10 & 178 & 27_1 & 107 \\
\hline $27 \_2$ & 110 & 27_3 & 142 & 27_4 & 105 & $27 \_5$ & 106 & $27 \_6$ & 133 & $27 \_7$ & 119 & $27 \_8$ & 136 \\
\hline 27_9 & 121 & 27_10 & 111 & 28_1 & 106 & 31_1 & 181 & 31_2 & 176 & 31_3 & 160 & 31_4 & 195 \\
\hline 31_5 & 187 & 31_6 & 182 & 31_7 & 191 & 31_8 & 176 & 31_99 & 176 & 31_10 & 202 & $32 \_1$ & 144 \\
\hline 32_2 & 123 & 32_5 & 133 & 32_6 & 122 & $32 \_8$ & 132 & 33_1 & 105 & 33_2 & 107 & 33_3 & 102 \\
\hline 33_4 & 107 & 33_8 & 107 & 33_9 & 109 & 34_1 & 76 & $34 \_2$ & 103 & 34_3 & 99 & $34 \_5$ & 102 \\
\hline 36_1 & 201 & 36_3 & 218 & 36_5 & 213 & 36_7 & 196 & 36_9 & 203 & 37_2 & 141 & $37 \_5$ & 195 \\
\hline 37_8 & 169 & 37_9 & 138 & 38_1 & 105 & $38 \_2$ & 119 & 38_4 & 138 & 38_6 & 119 & 38_7 & 103 \\
\hline 38_10 & 137 & 39_2 & 105 & 40_1 & 80 & 42_1 & 107 & 46_1 & 172 & 46_2 & 187 & 46_3 & 163 \\
\hline 46_5 & 136 & 46_7 & 158 & 46_9 & 157 & 46_10 & 175 & 47_1 & 130 & $47 \_3$ & 119 & $47 \_4$ & 120 \\
\hline $47 \_5$ & 126 & 47_6 & 128 & $47 \_7$ & 114 & 47_8 & 124 & 47_10 & 128 & 48_4 & 123 & 51_1 & 186 \\
\hline 51_2 & 200 & 51_3 & 193 & 51_4 & 197 & 51_6 & 193 & 51_7 & 185 & 51_8 & 186 & 51_99 & 190 \\
\hline 51_10 & 201 & 52_1 & 161 & $52 \_2$ & 169 & $52 \_3$ & 126 & $52 \_4$ & 157 & $52 \_5$ & 158 & $52 \_6$ & 183 \\
\hline $52 \_7$ & 142 & $52 \_8$ & 148 & 52_9 & 142 & 52_10 & 131 & 53_1 & 138 & 53_2 & 109 & 53_4 & 138 \\
\hline 53_5 & 109 & 53_6 & 101 & 53_8 & 135 & 53_10 & 124 & 54_1 & 102 & 54_5 & 107 & 54_6 & 104 \\
\hline 54_8 & 100 & 54_9 & 105 & 57_1 & 173 & $57 \_2$ & 151 & $57 \_3$ & 176 & $57 \_5$ & 170 & $57 \_6$ & 176 \\
\hline $57 \_7$ & 156 & 57_9 & 157 & 58_2 & 122 & 58_3 & 117 & 58_4 & 138 & 58_5 & 116 & 58_6 & 135 \\
\hline 58_7 & 143 & 58_8 & 126 & 58_9 & 126 & 59_5 & 104 & 59_6 & 112 & 59_8 & 107 & 59_99 & 117 \\
\hline 59_10 & 128 & 60_3 & 88 & 60_7 & 91 & & & & & & & & \\
\hline
\end{tabular}


Table 9: Lower and upper bounds for AT.

\begin{tabular}{|c|c|c|c|c|c|c|c|c|c|}
\hline inst & LB/UB & inst & LB/UB & inst & $\mathrm{LB} / \mathrm{UB}$ & inst & LB/UB & inst & $\mathrm{LB} / \mathrm{UB}$ \\
\hline $27 \_1$ & 41 & $27 \_2$ & 53 & $27 \_3$ & 68 & $27 \_4$ & $112 / 114$ & $27 \_5$ & 56 \\
\hline $27 \_6$ & 73 & $27 \_7$ & 54 & $27 \_8$ & 95 & 27_9 & 38 & 27_10 & 45 \\
\hline 27_11 & 57 & $27 \_12$ & 73 & $27 \_13$ & 38 & $27 \_14$ & 55 & $27 \_15$ & 46 \\
\hline 27_16 & 75 & 27_17 & 55 & 27_18 & 55 & 27_19 & 79 & 27_20 & 152 \\
\hline $27 \_21$ & 92 & $27 \_22$ & 86 & $27 \_23$ & 82 & $27 \_24$ & 106 & $27 \_25$ & 51 \\
\hline $27 \_26$ & 53 & $27 \_27$ & 58 & 27_28 & 95 & 27_29 & 51 & 27_30 & 76 \\
\hline 27_31 & 75 & $27 \_32$ & 82 & 27_33 & 66 & $27 \_34$ & 61 & 27_35 & 115 \\
\hline 27_36 & 146 & 27_37 & 78 & 27_38 & 100 & 27_39 & 119 & $27 \_40$ & 130 \\
\hline 27_41 & 60 & $27 \_42$ & 53 & $27 \_43$ & 75 & $27 \_44$ & 88 & $27 \_45$ & 49 \\
\hline $27 \_46$ & 65 & $27 \_47$ & 75 & $27 \_48$ & 80 & $51 \_1$ & 98 & 51_2 & 96 \\
\hline 51_3 & 133 & $51 \_4$ & $161 / 219$ & $51 \_5$ & 97 & $51 \_6$ & 126 & $51_{-} 7$ & 120 \\
\hline $51 \_8$ & 194 & 51_9 & 74 & 51_10 & 73 & 51_11 & 99 & 51_12 & $116 / 137$ \\
\hline 51_13 & 84 & $51 \_14$ & 86 & 51_15 & 86 & 51_16 & 132 & 51_17 & 84 \\
\hline 51_18 & 99 & _.19 & 170 & $1 \_20$ & 274 & 51_21 & 145 & 51_22 & 168 \\
\hline 51_23 & 183 & 51_24 & 228 & 51_25 & 95 & 51_26 & 89 & 51_27 & 113 \\
\hline 51_28 & 164 & 29 & 98 & & 105 & 51_31 & 130 & 51_32 & 139 \\
\hline 51_33 & 116 & 51_34 & 115 & 51_35 & 173 & 51_36 & 300 & 51_37 & 162 \\
\hline 51_38 & 177 & 51_39 & 189 & $51 \_40$ & 218 & $51 \_41$ & 102 & 51_42 & 108 \\
\hline 51_43 & 121 & $51 \_44$ & 174 & 51_45 & 122 & $51 \_46$ & 125 & $51 \_47$ & 151 \\
\hline 51_48 & 167 & 103_1 & 158 & 103_2 & 182 & 103_3 & $216 / 259$ & 103_4 & $280 / 445$ \\
\hline $103 \_5$ & 19 & $103 \_6$ & $207 / 209$ & $103 \_7$ & $234 / 293$ & $103 \_8$ & $207 / 294$ & 103_9 & 139 \\
\hline 103_10 & 119 & 103_11 & $160 / 169$ & 103_12 & $213 / 302$ & 103_13 & 127 & 103_14 & 152 \\
\hline 103_15 & $157 / 168$ & 103_16 & $167 / 179$ & 103_17 & 209 & 103_18 & 232 & 103_19 & 301 \\
\hline 103_20 & 475 & 103_21 & 276 & 103_22 & 295 & 103_23 & 368 & 103_24 & 449 \\
\hline 103_25 & 177 & 103_26 & 183 & 103_27 & 199 & 103_28 & 295 & 103_29 & 225 \\
\hline 103_30 & 231 & 103_31 & 227 & 103_32 & 281 & 103_33 & 220 & 103_34 & 264 \\
\hline 103_35 & 341 & 103_36 & 575 & 103_37 & 327 & 103_38 & 376 & 103_39 & 389 \\
\hline 103_40 & 451 & 103_41 & 191 & 103_42 & 187 & 103_43 & 260 & 103_44 & 375 \\
\hline 103_45 & 216 & $103 \_46$ & 251 & 103_47 & 262 & 103_48 & 300 & & \\
\hline
\end{tabular}

Table 10: Lower and upper bounds for PACK.

inst $\mathrm{LB} / \mathrm{UB}$ inst $\mathrm{LB} / \mathrm{UB}$ inst $\mathrm{LB} / \mathrm{UB}$ inst $\mathrm{LB} / \mathrm{UB}$ inst $\mathrm{LB} / \mathrm{UB}$ inst $\mathrm{LB} / \mathrm{UB}$

\begin{tabular}{lc|cc|cc|cc|cc|cc}
\hline 001 & 23 & 002 & 32 & 003 & 29 & 004 & $43 / 44$ & 005 & 42 & 006 & 47 \\
007 & 41 & 008 & 44 & 009 & $57 / 72$ & 010 & 38 & 011 & 44 & 012 & 45 \\
013 & 36 & 014 & 45 & 015 & 43 & 016 & 63 & 017 & 62 & 018 & 60 \\
019 & 59 & 020 & 62 & 021 & 51 & 022 & 59 & 023 & 51 & 024 & 56 \\
025 & $69 / 70$ & 026 & 54 & 027 & 55 & 028 & 64 & 029 & 43 & 030 & 20 \\
031 & 70 & 032 & 80 & 033 & 78 & 034 & 73 & 035 & $73 / 77$ & 036 & $100 / 106$ \\
037 & $116 / 138$ & 038 & 86 & 039 & $99 / 111$ & 040 & $87 / 91$ & 041 & 27 & 042 & 29 \\
043 & 105 & 044 & 103 & 045 & $86 / 87$ & 046 & $110 / 128$ & 047 & $103 / 107$ & 048 & $76 / 77$ \\
049 & 29 & 050 & $94 / 109$ & 051 & 29 & 052 & 85 & 053 & $97 / 113$ & 054 & $92 / 100$ \\
055 & $91 / 97$ & & & & & & & & & &
\end{tabular}


Table 11: Lower and upper bounds for PACK_D.

\begin{tabular}{cc|cc|cc|cc|cc} 
inst & LB/UB & inst & LB/UB & inst & LB/UB & inst & LB/UB & inst & LB/UB \\
\hline 001 & 612 & 002 & $745 / 747$ & 003 & $624 / 625$ & 004 & 1381 & 005 & 983 \\
006 & 1119 & 007 & 1082 & 008 & 1274 & 009 & $1593 / 1951$ & 010 & 1216 \\
011 & 940 & 012 & $1234 / 1241$ & 013 & 829 & 014 & 1565 & 015 & 1198 \\
016 & $1783 / 1813$ & 017 & $1641 / 1651$ & 018 & $1462 / 1480$ & 019 & $1526 / 1542$ & 020 & 1661 \\
021 & 1606 & 022 & 1787 & 023 & 1092 & 024 & 1625 & 025 & $2061 / 2147$ \\
026 & 926 & 027 & $1789 / 1793$ & 028 & $1897 / 1962$ & 029 & 1233 & 030 & 597 \\
031 & 1949 & 032 & 2943 & 033 & 3390 & 034 & 2371 & 035 & 2305 \\
036 & $2175 / 2191$ & 037 & $3325 / 3614$ & 038 & 2180 & 039 & $2730 / 2734$ & 040 & 3024 \\
041 & 679 & 042 & 838 & 043 & 2439 & 044 & 3050 & 045 & 2712 \\
046 & $3243 / 3277$ & 047 & $2740 / 2745$ & 048 & 2446 & 049 & 675 & 050 & $2687 / 2716$ \\
051 & 838 & 052 & 2253 & 053 & 2521 & 054 & 2750 & 055 & 2628
\end{tabular}

